\title{
Final results from OPERA
}

\section{Gabriele Sirri*}

INFN Sezione di Bologna, 40127 Bologna, Italy

E-mail: gabriele.sirri@bo.infn.it

\begin{abstract}
OPERA was a long-baseline experiment located at the Gran Sasso Laboratory and designed to search for $v_{\mu} \rightarrow v_{\tau}$ oscillations in the CERN-to-Gran Sasso neutrino beam. We report on the final analysis of the full data sample based on looser selection criteria than in previous analyses and multivariate approach. Oscillation parameters have been determined with a reduced statistical uncertainty, and the discovery of tau neutrino appearance is confirmed with an improved significance level. Moreover, the search for electron neutrino events has been extended to the full dataset exploiting an improved method for the electron neutrino energy estimation. New limits have been set in the framework of the $3+1$ neutrino model. Results on non-oscillation physics are also reported.
\end{abstract}

Neutrino Oscillation Workshop (NOW2018)

9 - 16 September, 2018

Rosa Marina (Ostuni, Brindisi, Italy)

*on behalf of the OPERA Collaboration 


\section{The OPERA experiment}

The OPERA experiment [1] was designed to study $v_{\mu} \rightarrow v_{\tau}$ oscillations in appearance mode. The detector was located in the underground Gran Sasso Laboratory (LNGS) and exposed to the CERN to Gran Sasso (CNGS) muon neutrino beam produced at a distance of $730 \mathrm{~km}$. The average neutrino energy was about $17 \mathrm{GeV}$, the $\bar{v}_{\mu}$ fraction was $2.1 \%$ in terms of expected CC interactions, the sum of $v_{e}$ and $\bar{v}_{e}$ was below $1 \%$, while the prompt $v_{\tau}$ contamination was negligible.

The detector was a hybrid apparatus made of an emulsion and lead target with a total mass of about $1.25 \mathrm{kt}$, complemented by electronic detectors. The general structure consisted of two identical supermodules (SM). Each SM was made of a target section, composed of 31 target walls, and a muon spectrometer. Each target wall was an assembly of horizontal trays loaded with target units, called bricks. Each brick consisted of 57 emulsion films, $300 \mu \mathrm{m}$ thick, interleaved with 56 lead plates, $1 \mathrm{~mm}$ thick. Bricks had a cross section of $12.7 \times 10.2 \mathrm{~cm}^{2}$ and a thickness of 7.5 $\mathrm{cm}$ corresponding to about ten radiation lengths and a mass of $8.3 \mathrm{~kg}$. Downstream of each target wall, two orthogonal planes of scintillator strips (target tracker detector) recorded the position and the energy deposition of charged particles. Finally, a magnetic spectrometer instrumented with resistive plate chambers and high-resolution drift tubes was used to identify muons and measure their charge and momentum [1]. A sample corresponding to $17.97 \cdot 10^{19}$ protons on target has been collected from 2008 to 2012 and resulted in 19505 neutrino interactions in the target fiducial volume.

\section{Results on $v_{\mu} \rightarrow v_{\tau}$ oscillation search}

The appearance of the $v_{\tau}$ was identified by the detection of characteristic decay topologies of the tau lepton produced in CC interactions. Kinematical selection criteria were applied to reduce the background arising from processes that mimic the $\tau$ decay topologies, which are (i) decays of charmed particles produced in $v_{\mu}$ CC interactions; (ii) reinteractions of hadrons from $v_{\mu}$ events occurring in lead plates; (iii) large-angle scattering of muons produced in $v_{\mu} \mathrm{CC}$ interactions. In 2015 , five $v_{\tau}$ candidates were reported with an expected background of $0.25 \pm 0.05$ events. The discovery of $v_{\mu} \rightarrow v_{\tau}$ oscillations was assessed with a significance of 5.1 $\sigma$ [2].

In 2018 a new analysis based on a multivariate approach for event identification, fully exploiting the expected features of $v_{\tau}$ events was performed [3]. It was applied on candidate events selected with looser criteria than those applied in the previous analysis. Ten tau neutrino candidates were identified in the full OPERA dataset with an expected background of $2.0 \pm 0.4$ events. The discovery significance increased to $6.1 \sigma$.

Assuming maximal mixing and $v_{\tau} \mathrm{CC}$ interaction cross section as in default implementation provided by GENIE v2.6, an interval of $\left|\Delta m_{32}^{2}\right|=\left(2.7_{0.6}^{+0.7}\right) \cdot 10^{3} \mathrm{eV}^{2}$ was derived at $68 \%$ C.L. using the Feldman-Cousins method. This is the first estimate of $\left|\Delta m_{32}^{2}\right|$ in appearance mode and it is consistent with results from disappearance experiments, including the global best-fit value [4]. Alternatively, by fixing $\left|\Delta m_{32}^{2}\right|$ at the global best-fit value $\left(2.50 \cdot 10^{3} \mathrm{eV}^{2}\right)$ and maximal mixing, $\sin ^{2} 2 \theta_{23}=1$, one can estimate the $v_{\tau} \mathrm{CC}$ cross section on the lead target [5]. The total flux integrated cross section is $\langle\sigma\rangle_{\text {meas }}=5.1_{2.0}^{+2.4} \cdot 10^{-36} \mathrm{~cm}^{2}$. 


\section{Results on $v_{\mu} \rightarrow v_{e}$ oscillation search}

The OPERA nuclear emulsion granularity allowed the reconstruction of electromagnetic showers and thus the identification of $v_{e} \mathrm{CC}$ interactions. A dedicated procedure [6], balancing computation time and detection efficiency, was defined to systematically search for $v_{e}$ events. A total of $35 v_{e}$ events were observed. The number is compatible with what expected from $v_{e}$ beam contamination, $(30.7 \pm 3.2)$ events, together with two main sources of background: i) $\pi^{0}$ 's misidentified as electrons in neutrino interactions without a reconstructed muon, $(0.5 \pm 0.5)$ events and ii) $v_{\tau} \mathrm{CC}$ interactions with $\tau$ decaying into an electron, $(0.7 \pm 0.2)$ events. Using the global best-fit values [4] for $\theta_{13}, \theta_{23}$ and $\Delta m_{\text {atm }}^{2}$, assuming $\delta_{C P}=0$ and neglecting matter effects, a total of $34.3 \pm 3.5$ $v_{e}$ candidate events were expected in the whole energy range. The number of observed events is compatible with the three flavors oscillation model and an upper limit on $\sin ^{2} 2 \theta_{13}<0.43$ was set at $90 \%$ C.L. in the [0-40] GeV neutrino energy range.

\section{Results on sterile neutrino search in the $(3+1)$ neutrino model}

In presence of a fourth sterile neutrino ( $3+1$ model), the oscillation probability is a function of a $4 \times 4$ mixing matrix $U$ and of three squared mass differences. The $(3+1)$ neutrino flux prediction at OPERA was simulated using GLoBES. The parameter $\Delta m_{21}^{2}$ was fixed to the global best-fit value [4], a Gaussian external constraint on $\Delta m_{31}^{2}$ was set using mean and sigma from a global fit [4], while only positive values of $\Delta m_{41}^{2}$ were considered. Matter effects were taken into account with the Earth density approximated by a constant value estimated with the PREM [7] onion shell model. The systematic uncertainty on the expected number of tau neutrino candidates is conservatively set to $20 \%$, largely dominated by the limited knowledge of $v_{\tau}$ interaction cross section and the detection efficiency. The systematic error on the expected number of electron neutrino candidates, due to beam normalization and efficiency uncertainties, is $10 \%$ (20\%) for events with neutrino energy larger (smaller) than $10 \mathrm{GeV}$.

OPERA can fit simultaneously tau and electron neutrino data (i.e. the visible energy of $v_{\tau}$ events and the reconstructed energy of $v_{e}$ events) to constrain sterile neutrino oscilation parameters. Excluded regions on $\sin ^{2} 2 \theta_{\mu \tau}=4\left|U_{\mu 4}\right|^{2}\left|U_{\tau 4}\right|^{2}$ vs $\Delta m_{41}^{2}$ and $\sin ^{2} 2 \theta_{\mu e}=4\left|U_{\mu 4}\right|^{2}\left|U_{e 4}\right|^{2}$ vs $\Delta m_{41}^{2}$ planes are preliminarily estimated. For $\Delta m_{41}^{2} \gtrsim 1 \mathrm{eV}^{2}$, the $90 \%$ C.L. upper limit on $\sin ^{2} 2 \theta_{\mu \tau}$ is 0.099. At large $\left|\Delta m_{41}^{2}\right|$ values, the $90 \%$ C.L. upper limit on $\sin ^{2} 2 \theta_{\mu e}$ is 0.019 .

\section{Results from non-oscillation physics search}

The characteristics of the multiplicity distribution of charged hadrons in neutrino-lead interactions can be helpful in tuning the models implemented in $\mathrm{MC}$ event generators. For this porpouse a sub-sample of about 800 events with a muon in the final state identified by the muon spectrometers was analyzed. Quasi-elastic interactions are rejected by selecting events with a square of the invariant mass of the hadronic system $W^{2}>1 \mathrm{GeV}^{2} / \mathrm{c}^{4}$. Fragments from the target nuclei and recoil nuclei are excluded from the hadron shower multiplicity calculation thanks to their high ionizing power measured with emulsions. Results can be summarized as follow [8]: i) the dependence of the average multiplicity $<n_{c h}>$ on $\ln W^{2}$ is approximately linear; ii) the dependence of the dispersion 
$D_{c h}=\sqrt{\left.<n_{c h}^{2}>-<n_{c h}\right\rangle^{2}}$ on $<n_{c h}>$ is also approximately linear; iii) the approximate KobaNielsen-Olesen (KNO) scaling is valid for the charged hadrons multiplicity, i.e., the multiplicity distribution is independent of the primary energy.

OPERA electronic systems, i.e., the target trackers and the muon spectrometers, were used to detect muons produced by interactions of primary cosmic rays in the upper atmosphere. The overburden of the Gran Sasso underground laboratory, $3800 \mathrm{~m}$ water equivalent, selects muons of $\mathrm{TeV}$ energy. Using single cosmic muon events, OPERA measured the charge ratio $\left(R=N_{\mu+} / N_{\mu-}\right)$ [9] and the annual modulation of the flux [10].

The flux of single cosmic ray muons in underground laboratories shows an annual modulation as a consequence of the atmospheric temperature variation which, changing the air density, modifies the proportion between $\pi / K$ mesons decays (into muons) and interactions. The amplitude of the muon flux modulation amounts to $(1.55 \pm 0.08) \%$ of the average value. The relative variations of the muon flux and of the effective atmospheric temperature are correlated with a constant $\alpha_{T}$, depending on the average energy of the muons reaching the underground detectors. With the muons reconstructed by the OPERA electronic detectors, a value $\alpha_{T}=(0.95 \pm 0.04)$ has been estimated for 1.1 TeV muons, consistent with other LNGS experiments.

\section{References}

[1] R. Acquafredda et al., The OPERA experiment in the CERN to Gran Sasso neutrino beam, JINST 4 (2009) P04018.

[2] N. Agafonova et al., Discovery of tau Neutrino Appearance in the CNGS Neutrino Beam with the OPERA Experiment, Phys. Rev. Lett. 115 (2015) 121802 [arXiv:1507.01417 [hep-ex] ] .

[3] N. Agafonova et al., Final Results of the OPERA Experiment on $v_{\tau}$ Appearance in the CNGS Neutrino Beam, Phys. Rev. Lett. 120 (2018) 211801, Erratum: Phys. Rev. Lett. 121 (2018) 139901 [arXiv:1804.04912 [hep-ex]].

[4] M. Tanabashi et al., Review of Particle Physics, Phys. Rev. D 98 (2018) no.3, 030001.

[5] A. Anokhina et al., Study of the effects induced by lead on the emulsion films of the OPERA experiment, JINST 3 (2008) P07002 [arXiv:0805.0123 [physics.ins-det] ] .

[6] N. Agafonova et al., Search for $v_{\mu} \rightarrow v_{e}$ oscillations with the OPERA experiment in the CNGS beam, JHEP 1307 (2013) 004, Addendum: JHEP 1307 (2013) 085 [arXiv: 1303.3953 [hep-ex] ].

[7] P. Huber et al., New features in the simulation of neutrino oscillation experiments with GLoBES 3.0: General Long Baseline Experiment Simulator, Comput. Phys. Commun. 177 (2007) 432 [hep-ph/0701187].

[8] N. Agafonova et al., Study of charged hadron multiplicities in charged-current neutrino-lead interactions in the OPERA detector, Eur. Phys. J. C 78 (2018) 62 [arXiv: 1706.07930 [hep-ex] ].

[9] N. Agafonova et al., Measurement of the TeV atmospheric muon charge ratio with the complete OPERA data set, Eur. Phys. J. C 74 (2014) 2933 [arXiv: 1403.0244 [hep-ex] ] .

[10] N. Agafonova et al., Measurement of the cosmic ray muon flux seasonal variation with the OPERA detector, submitted to JCAP, arXiv:1810.10783 [hep-ex] . 